2004-01-01

\title{
Riemann Tensor of the Ambient Universe, the Dilaton, and Newton's Constant
}

\author{
Rossen Ivanov \\ Technological University Dublin, rossen.ivanov@tudublin.ie \\ Emil Prodanov \\ Technological University Dublin, emil.prodanov@tudublin.ie
}

Follow this and additional works at: https://arrow.tudublin.ie/scschmatart

Part of the Cosmology, Relativity, and Gravity Commons

\section{Recommended Citation}

Ivanov, R. \& Prodanov, E. (2004). Riemann Tensor of the Ambient Universe, the Dilaton and the Newton's Constant. Physical Review D, vol. 70, no. 4. doi:10.1103/PhysRevD.70.044013

This Article is brought to you for free and open access by the School of Mathematics at ARROW@TU Dublin. It has been accepted for inclusion in Articles by an authorized administrator of ARROW@TU Dublin. For more information, please contact arrow.admin@tudublin.ie, aisling.coyne@tudublin.ie,gerard.connolly@tudublin.ie.

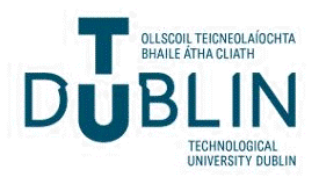




\title{
Riemann tensor of the ambient universe, the dilaton, and Newton's constant
}

\author{
Rossen I. Ivanov* \\ Institute for Nuclear Research and Nuclear Energy, Bulgarian Academy of Sciences, 72 Tsarigradsko chaussee, Sofia 1784, Bulgaria \\ and Faculty of Engineering and Computing, Dublin City University, Dublin, Ireland \\ Emil M. Prodanov ${ }^{\dagger}$ \\ School of Physical Sciences, Dublin City University, Dublin, Ireland
}

(Received 25 September 2003; published 13 August 2004)

\begin{abstract}
We investigate a four-dimensional world, embedded into a five-dimensional spacetime, and find the fivedimensional Riemann tensor via generalization of the Gauss(-Codacci) equations. We then derive the generalized equations of the four-dimensional world and also show that the square of the dilaton field is equal to Newton's constant. We find plausible constant and nonconstant solutions for the dilaton.
\end{abstract}

DOI: 10.1103/PhysRevD.70.044013

PACS number(s): 04.50.+h, 04.20.Cv, 98.80.-k

Since the pioneering work of Kaluza [1] and Klein [2], who unified gravitation with electromagnetism, the implications of possible extra dimensions to our world have been under intense investigation-see [3] for an extensive collection of papers on higher-dimensional unification. Jordan and Müller [4] and Thiry [5] used the equations of Kaluza-Klein theory to show that the gravitational constant can be expressed as a dynamical field. A constant solution for Newton's constant, however, is then allowed only if the square of the Maxwell electromagnetic tensor vanishes. Here we examine a dual setup in which this problem can be avoided. Based on the original Kaluza model, we are here considering a general embedding of a four-dimensional world into a fivedimensional ambient spacetime. We derive a generalization of the Gauss(-Codacci) equations by utilizing all degrees of freedom (the entire geometry) of the ambient spacetime, and we also show how they affect the physics of the fourdimensional world. As a result we find a system of equations for the electromagnetic field, gravitational field, and dilaton field. One of these equations is a plausible generalization of the gauge fixed Maxwell equations in the presence of a dilaton field. We also show that the square of the dilaton field is equal to (modulo numerical factors) the Newton's constant. The gauge freedom of the electromagnetic fields is transferred to a freedom in fixing the dilaton field. Apart from the constant solution for the dilaton, we give an example of a nonconstant solution describing a time-varying Newton constant in an expanding universe (see also [6-11] and others). We also give a general formula for generating different solutions for the dilaton field.

We consider a four-dimensional world $M$, embedded into a five-dimensional spacetime $V$ (see [12-22] and references therein for a detailed discussion on embeddings). Let $y^{i}(i$ $=1,2,3,4)$ denote the coordinates on $M$ and $x^{\mu}(\mu$ $=1,2,3,4,5)$ denote the coordinates on $V$. Greek indices will be related to the five-dimensional spacetime $V$, while latin indices will be associated with the four-dimensional spacetime $M$. Let $\psi\left(x^{\mu}\right)=s=$ const be the equation of the hyper-

\footnotetext{
*Electronic address: rossen.ivanov@dcu.ie

†Electronic address: prodanov@physics.dcu.ie
}

surface $M$. We assume that this equation is given a priori. The physical principles motivating the choice of type of hypersurface [specified by the function $\psi\left(x^{\mu}\right)$ ] and the choice of a particular four-dimensional spacetime slice (given by $s$ ) are open problems for multidimensional theories and not the subject of our analysis. We will retain $s$ as a parameter of the model.

One can alternatively express the parametric equations of $M$ as $x^{\mu}=x^{\mu}\left(y^{j}, s\right)$ and treat the parameter $s$ as a coordinate; this then represents a coordinate transformation with inverse

$$
y^{j}=y^{j}\left(x^{\mu}\right), \quad s=\psi\left(x^{\mu}\right) .
$$

We assume that this transformation is invertible at each point. This means that the Jacobi matrices of the transformation and its inverse have nonvanishing determinants everywhere. Thus, to globally parametrize the foliated fivedimensional spacetime $V$, it is sufficient to use the coordinates of the four-dimensional world $M$ and the foliation parameter $s$.

The vector normal to the surface is

$$
N_{\mu}=\frac{\partial \psi}{\partial x^{\mu}}
$$

Let us also define

$$
e_{j}^{\mu}=\frac{\partial x^{\mu}}{\partial y^{j}}, \quad n^{\mu}=\frac{\partial x^{\mu}}{\partial s}, \quad E_{\mu}^{i}=\frac{\partial y^{i}}{\partial x^{\mu}} .
$$

The derivatives are related as follows:

$$
\begin{aligned}
& \partial_{k} \equiv \frac{\partial}{\partial y^{k}}=e_{k}^{\mu} \partial_{\mu}, \\
& \partial_{\nu} \equiv \frac{\partial}{\partial x^{\nu}}=E_{\nu}^{k} \partial_{k}+N_{\nu} \partial_{s} .
\end{aligned}
$$

Obviously, if we denote $e_{5}^{\mu}=n^{\mu}$ and $E_{\mu}^{5}=N_{\mu}$, then $\left(e_{\nu}^{\mu}\right)$ and $\left(E_{\nu}^{\mu}\right)$ will be the Jacobi matrices of the transformation $\left(x^{\mu}\right)$ $\rightarrow\left(y^{i}, s\right)$ and its inverse. Therefore, 


$$
e_{\sigma}^{\mu} E_{\nu}^{\sigma}=\delta_{\nu}^{\mu}=E_{\sigma}^{\mu} e_{\nu}^{\sigma}
$$

This orthogonality condition is equivalent to

$$
\begin{aligned}
e_{i}^{\mu} E_{\mu}^{j} & =\delta_{i}^{j}, \\
N_{\mu} n^{\mu} & =1, \\
E_{\mu}^{i} e_{i}^{\nu}+N_{\mu} n^{\nu} & =\delta_{\mu}^{\nu}, \\
N_{\mu} e_{j}^{\mu} & =0, \\
n^{\mu} E_{\mu}^{j} & =0 .
\end{aligned}
$$

(Note that $\delta_{\mu}^{\mu}=\operatorname{dim} V=5$ and $\delta_{i}^{i}=\operatorname{dim} M=4$.)

Thus the bases $\left(e_{j}^{\mu}, n^{\mu}\right)$ and $\left(E_{\mu}^{j}, N_{\mu}\right)$ are dual. They do not depend on the metric of either spacetime, but only on the particular embedding chosen.

Let us now introduce a scalar field $\phi\left(y^{k}, s\right)$, a vector field $A_{i}\left(y^{k}, s\right)$, and the metric tensor $g_{i j}\left(y^{k}, s\right)$, which are $M$-valued tensor functions on $V$. We further define the metric $G_{\mu \nu}$ of the five-dimensional spacetime $V$ as an expansion over the basis vectors $E_{\mu}^{i}$ and $N_{\mu}$ :

$$
G_{\mu \nu}=E_{\mu}^{i} E_{\nu}^{j} g_{i j}+\left(N_{\mu} E_{\nu}^{i}+N_{\nu} E_{\mu}^{i}\right) A_{i}+N_{\mu} N_{\nu} \phi .
$$

Taking $x^{i}=y^{i}, x^{5}=s=$ const, i.e., $\quad e_{i}^{\mu}=\delta_{i}^{\mu}, n^{\mu}=\delta_{5}^{\mu}, E_{\mu}^{i}$ $=\delta_{\mu}^{i}, N_{\mu}=\delta_{\mu}^{5}$ in Eq. (12) corresponds to the original Kaluza model [1]. Klein's modification [2] $g_{i j} \rightarrow g_{i j}+A_{i} A_{j}$, together with the identification of $\phi$ as a dilaton is the model put forward by Jordan and Müller [4] and Thiry [5]. We note that the metric (12) has the same form as the inverse of the metric of Klein's model, and thus the two theories are dual: Kaluza's model corresponds to slicing, while Klein's model corresponds to threading of the five-dimensional spacetime [15]. The case with $A_{i}=0$ has also been considered (see, for example, [22,23] and references therein).

The lack of gauge invariance for the fields $A_{i}$, which we nevertheless will associate with the electromagnetic potentials, in view of the slicing-threading duality, is compensated by the freedom to fix $\phi$. This, as will become clear later, is the freedom to fix the dilaton field.

Returning to Eq. (12), we define $g^{i j}$ as the inverse of the metric $g_{i j}$. Thus $A^{i}=g^{i j} A_{j}$ and $A^{2}=g^{i j} A_{i} A_{j}$.

The inverse $G^{\mu \nu}$ of the metric $G_{\mu \nu}$ on $V$ is then given by

$$
G^{\mu \nu}=h^{i j} e_{i}^{\mu} e_{j}^{\nu}-\theta A^{i}\left(e_{i}^{\mu} n^{\nu}+e_{i}^{\nu} n^{\mu}\right)+\theta n^{\mu} n^{\nu},
$$

where $\theta=\left(\phi-A^{2}\right)^{-1}$ and $h^{i j}=g^{i j}+\theta A^{i} A^{j}$. One can easily check that $G^{\mu \lambda} G_{\lambda \nu}=\delta_{\nu}^{\mu}$. Using the inverse $G^{\mu \nu}$ (13) of the metric $G_{\mu \nu}$, we can raise and lower the five-dimensional indices to get

$$
\begin{aligned}
& N^{\mu}=G^{\mu \nu} N_{\nu}=\theta\left(n^{\mu}-A^{i} e_{i}^{\mu}\right), \\
& N^{2}=N_{\mu} N^{\mu}=\theta, \\
& n_{\mu}=G_{\mu \nu} n^{\nu}=A_{i} E_{\mu}^{i}+N_{\mu} \phi, \\
& n^{2}=n_{\mu} n^{\mu}=\phi .
\end{aligned}
$$

Note that when $A^{i}=0$ and $\phi=1$, then $N^{\mu}=n^{\mu}$ as in the Arnowitt-Deser-Misner approach [22].

The extrinsic curvature is

$$
K_{j l}=e_{j}^{\mu} e_{l}^{\nu} Q_{\mu \nu},
$$

where $Q_{\mu \nu}=\nabla_{\mu} N_{\nu}=\nabla_{\nu} N_{\mu}$.

Multiplying Eq. (18) across by $E_{\alpha}^{j} E_{\beta}^{l}$ and applying the orthogonality conditions $(7)-(11)$, one easily finds

$$
Q_{\alpha \beta}=E_{\alpha}^{i} E_{\beta}^{j} K_{i j}+\left(N_{\alpha} E_{\beta}^{i}+N_{\beta} E_{\alpha}^{i}\right) f_{i}+N_{\alpha} N_{\beta} \chi,
$$

with $\chi=n^{\mu} n^{\nu} Q_{\mu \nu}$ and

$$
f_{j}=n^{\mu} e_{j}^{\beta} Q_{\mu \beta}=A^{i} K_{i j}+\frac{1}{N} \partial_{j} N,
$$

where $N=\sqrt{N_{\mu} N^{\mu}}$.

The four-dimensional Christoffel symbols

$$
\gamma_{j k}^{i}=\frac{1}{2} g^{i l}\left(\partial_{k} g_{l j}+\partial_{j} g_{l k}-\partial_{l} g_{j k}\right)
$$

can then be expressed as

$$
\gamma_{j l}^{i}=\left(\partial_{j} e_{l}^{\mu}+e_{j}^{\alpha} e_{l}^{\beta} \Gamma_{\alpha \beta}^{\mu}\right) E_{\mu}^{i}-K_{j l} A^{i},
$$

where $\Gamma_{\alpha \beta}^{\mu}$ are the five-dimensional Christoffel symbols. Further, the four-dimensional Riemann curvature tensor

$$
r_{j k l}^{i}=\partial_{k} \gamma_{j l}^{i}-\partial_{l} \gamma_{j k}^{i}+\gamma_{j l}^{m} \gamma_{m k}^{i}-\gamma_{j k}^{m} \gamma_{m l}^{i},
$$

using Eqs. (22) and (7)-(10), becomes

$$
\begin{aligned}
r_{j k l}^{i}= & E_{\alpha}^{i} e_{j}^{\lambda} e_{k}^{\mu} e_{l}^{\nu} R_{\lambda \mu \nu}^{\alpha}+E_{\alpha}^{i}\left(\nabla_{\mu} n^{\alpha}\right)\left(e_{k}^{\mu} K_{l j}-e_{l}^{\mu} K_{k j}\right) \\
& +\nabla_{l}\left(A^{i} K_{k j}\right)-\nabla_{k}\left(A^{i} K_{l j}\right)+A^{i} A^{m}\left(K_{m l} K_{k j}-K_{m k} K_{l j}\right)
\end{aligned}
$$

where $R_{\lambda \mu \nu}^{\alpha}$ is the five-dimensional Riemann curvature tensor. The above is a generalization of the Gauss equations. Taking $A^{i}=0$ and $\phi=1$, one simply recovers the well known Gauss equations (see, for example, [24])

$$
r_{j k l}^{i}=E_{\alpha}^{i} e_{j}^{\lambda} e_{k}^{\mu} e_{l}^{\nu} R_{\lambda \mu \nu}^{\alpha}+K_{k}^{i} K_{l j}-K_{l}^{i} K_{k j} .
$$

Let us now expand the five-dimensional Riemann curvature tensor over our basis. Using its symmetries we can write

$$
\begin{aligned}
R_{\lambda \mu \nu \sigma}= & E_{\lambda}^{i} E_{\mu}^{j} E_{\nu}^{k} E_{\sigma}^{l} U_{i j k l}+\left[\left(N_{\lambda} E_{\mu}^{j}-N_{\mu} E_{\lambda}^{j}\right) E_{\nu}^{k} E_{\sigma}^{l}\right. \\
& \left.+\left(N_{\nu} E_{\sigma}^{j}-N_{\sigma} E_{\nu}^{j}\right) E_{\lambda}^{k} E_{\mu}^{l}\right] V_{j k l} \\
& +\left(N_{\lambda} E_{\mu}^{j}-N_{\mu} E_{\lambda}^{j}\right)\left(N_{\nu} E_{\sigma}^{l}-N_{\sigma} E_{\nu}^{l}\right) W_{j l},
\end{aligned}
$$

where the coefficients in this expansion satisfy

$$
\begin{gathered}
U_{i j k l}=U_{k l i j}=-U_{i j l k}=-U_{j i k l}, \\
V_{j k l}=-V_{j l k}, \quad W_{j l}=W_{l j} .
\end{gathered}
$$

Using Eq. (24), one can further find 


$$
\begin{aligned}
U_{i j k l} & =e_{i}^{\lambda} e_{j}^{\mu} e_{k}^{\nu} e_{l}^{\sigma} R_{\lambda \mu \nu \sigma} \\
& =r_{i j k l}-\left(\pi_{i k} \pi_{l j}-\pi_{i l} \pi_{k j}\right), \\
V_{j k l} & =n^{\lambda} e_{j}^{\mu} e_{k}^{\nu} e_{l}^{\sigma} R_{\lambda \mu \nu \sigma} \\
& =A^{i} U_{i j k l}-\frac{1}{N}\left(\nabla_{k} \pi_{l j}-\nabla_{l} \pi_{k j}\right),
\end{aligned}
$$

where $\pi_{j l}=(1 / N) K_{j l}$.

Finding the remaining tensor $W_{j l}$ is more complicated. One can easily see that

$$
W_{j l}=n^{\mu} n^{\sigma} e_{j}^{\lambda} e_{l}^{\nu} R_{\lambda \mu \nu \sigma}=S_{j l}+A^{k} V_{l k j},
$$

where

$$
S_{j l}=\frac{1}{N^{2}} n^{\sigma} e_{j}^{\lambda} e_{l}^{\nu}\left(\nabla_{\lambda} Q_{\sigma \lambda}-\nabla_{\sigma} Q_{\nu \lambda}\right) .
$$

In the above we identify derivatives in the direction of $n^{\sigma}$. To handle this type of term, we will have to explicitly invoke the dependence on $s$.

First, by virtue of Eq. (5), we get the following expression for the extrinsic curvature (18):

$$
\begin{aligned}
K_{j l}= & -\frac{N^{2}}{2}\left(\nabla_{j} A_{l}+\nabla_{l} A_{j}\right)+\frac{N^{2}}{2} \partial_{s} g_{j l} \\
& -\frac{1}{2} N^{\lambda}\left(g_{k l} \partial_{j} E_{\lambda}^{k}+g_{k j} \partial_{l} E_{\lambda}^{k}+A_{l} \partial_{j} N_{\lambda}+A_{j} \partial_{l} N_{\lambda}\right) \\
& +\frac{N^{2}}{2} G_{\mu \nu}\left[A^{k} \partial_{k}\left(e_{j}^{\mu} e_{l}^{\nu}\right)-\partial_{s}\left(e_{j}^{\mu} e_{l}^{\nu}\right)\right] .
\end{aligned}
$$

Using Eqs. (20), (31), (32), and (33), it follows that

$$
\begin{aligned}
W_{j l}= & \frac{1}{N^{3}} \nabla_{j} \nabla_{l} N-\frac{2}{N^{4}}\left(\partial_{j} N\right)\left(\partial_{l} N\right)+A^{i} A^{k} U_{i j k l} \\
& +\frac{1}{N}\left[\nabla_{j}\left(A^{k} \pi_{k l}\right)+\nabla_{l}\left(A^{k} \pi_{k j}\right)\right]-\frac{1}{N} A^{k} \nabla_{k} \pi_{l j} \\
& +\frac{1}{N^{2}} \pi_{k l} \pi_{j}^{k}-\frac{1}{N} \partial_{s} \pi_{j l}+\Omega_{j l},
\end{aligned}
$$

where $\Omega_{j l}$ contains only terms that are proportional to derivatives of the basis vectors and their duals with respect to $\left(y^{k}, s\right)$.

The five-dimensional Ricci tensor can easily be calculated from Eq. (26):

$$
\begin{aligned}
R_{\mu \nu}= & E_{\mu}^{j} E_{\nu}^{l}\left[h^{i k} U_{i j k l}-N^{2} A^{k}\left(V_{j k l}+V_{l k j}\right)+N^{2} W_{j l}\right]+\left(N_{\mu} E_{\nu}^{l}\right. \\
& \left.+N_{\nu} E_{\mu}^{l}\right)\left(-h^{j k} V_{j k l}+N^{2} A^{j} W_{j l}\right)+N_{\mu} N_{\nu} h^{j l} W_{j l} .
\end{aligned}
$$

Then the five-dimensional Einstein equations in vacuum

$$
R_{\mu \nu}=0
$$

reduce to

$$
\begin{aligned}
h^{i k} U_{i j k l}-N^{2} A^{k}\left(V_{j k l}+V_{l k j}\right)+N^{2} W_{j l} & =0, \\
h^{j k} V_{j k l}-N^{2} A^{j} W_{j l} & =0, \\
h^{j l} W_{j l} & =0 .
\end{aligned}
$$

Multiplying Eq. (37) by $A^{j}$ and adding it to Eq. (38) allows us to exclude $W_{j l}$ from Eq. (38). Then, using the expressions (29) and (30) for $U_{i j k l}$ and $V_{j k l}$, Eq. (38) becomes

$$
\nabla_{k} \pi_{l}^{k}-\nabla_{l} \pi_{k}^{k}=0 \text {. }
$$

Equations (40) (as we will see below) are a generalization of Maxwell's equations in a fixed gauge.

One has to make a very important point here. Klein's theory corresponds to a threading decomposition of the fivedimensional spacetime [15]. Rigorous analysis [16] shows that the curvature tensor of the hypersurface formed is given by Zelmanov's curvature tensor, which differs from the ordinary Riemann curvature tensor by additional terms containing $s$ derivatives of the four-dimensional metric [17-21]. Independence of the parameter $s$ forces the two curvature tensors to be equal and thus represents a surface forming condition. In Kaluza's theory, the foliation of the fivedimensional spacetime corresponds to slicing [15]. Then the four-dimensional metric $g_{i j}$ naturally appears as the slicing metric, and imposing independence on the parameter $s$ is not at all necessary.

To simplify the analysis of the physics described by the fields $A_{i}, \phi$, and $N$, we will, however, put aside the $s$-dependent terms. Also, for simplicity, we will assume that the basis elements and their duals are constant (thus recovering the original Kaluza theory). The tensor $\Omega_{j l}$ will then vanish from Eq. (34).

Equation (40) becomes

$$
\nabla_{k} F^{k l}=-2 A_{k} r^{k l}+\frac{2}{N^{2}}\left(\pi^{k l}-\pi_{j}^{j} g^{k l}\right) \partial_{k} N .
$$

Here $F_{k l}=\nabla_{k} A_{l}-\nabla_{l} A_{k}$ is the Maxwell electromagnetic tensor with $A_{k}$ being the electromagnetic potential.

The first term on the right-hand side of Eq. (41) describes an interaction between electromagnetic and gravitational fields. We assume that it is much smaller than the remaining terms, so that we can neglect it. Note that $A_{k}$ cannot be "gauged up" to increase the scale of $A_{k} r^{k l}$. Furthermore, if $N$ is a constant, then Eq. (41) becomes the usual Maxwell equations

$$
\nabla_{k} F^{k l}=0 .
$$

The remaining two equations are

$$
\begin{gathered}
\nabla_{k}\left(\frac{f^{k}}{N}\right)=0, \\
r_{j l}-\frac{1}{2} g_{j l} r=\frac{N^{2}}{2} T_{j l},
\end{gathered}
$$

where $r=g^{i k} r_{i k}$ and $r_{j l}$ are the four-dimensional scalar curvature and four-dimensional Ricci tensor, respectively.

The energy-momentum tensor $T_{j l}$ is therefore given by 


$$
T_{j l}=T_{j l}^{\text {Maxwell }}+g^{i k} \nabla_{i} B_{j l k}+C_{j l}+D_{j l},
$$

where

$$
\begin{aligned}
T_{j l}^{\text {Maxwell }=} & g^{i k} F_{i j} F_{k l}-\frac{1}{4} g_{j l} F_{i k} F^{i k}, \\
B_{j l k}= & A_{k} \nabla_{l} A_{j}-A_{l} \nabla_{k} A_{j}-A_{j} F_{k l}+\nabla_{j}\left(A_{k} A_{l}\right) \\
& +g_{j l}\left(A^{i} \nabla_{k} A_{i}-A_{k} \nabla_{i} A^{i}\right), \\
C_{j l}= & g_{j l} A^{i} A^{k} r_{i k}-2 A^{i} A_{l} r_{i j}-2 A^{i} A_{j} r_{i l}, \\
D_{j l}= & \frac{4}{N^{4}}\left(\partial_{j} N\right)\left(\partial_{l} N\right)-\frac{2}{N^{3}} \nabla_{j} \nabla_{l} N \\
& -\frac{2}{N^{2}} \pi_{k}^{k}\left(A_{l} \partial_{j} N+A_{j} \partial_{l} N\right)+\frac{2}{N^{2}}\left[-A^{k} \pi_{j l}\right. \\
& \left.+A_{l} \pi_{j}^{k}+A_{j} \pi_{l}^{k}-g_{j l}\left(A^{i} \pi_{i}^{k}-A^{k} \pi_{i}^{i}\right)\right] \partial_{k} N .
\end{aligned}
$$

We will analyze each of these terms separately. The first one $T_{j l}^{\text {Maxwell }}$ is the Maxwell energy-momentum tensor. The tensor $C_{j l}$ describes the interaction between the electromagnetic and gravitational fields. From Eq. (44) we see that, if $N^{2}$ is very small (as we will confirm later), then $r_{j l}$ will be of the order of $N^{2}$, which justifies the neglect of the interaction terms in Eq. (41) and the tensor $C_{j l}$.

Using Eq. (33) in Eq. (20) and then Eq. (20) in Eq. (43), we see that a constant solution for $N$ is allowed by Eq. (43) if $\phi$ satisfies

$$
\nabla^{k} \partial_{k} \phi=\frac{1}{2} F^{i k} F_{i k}
$$

where $F^{i k}$ is a solution of Eq. (42). For the constant solution for $N$, the tensor $D_{j l}$ vanishes. Moreover,

$$
0 \equiv g^{i j} \nabla_{i} T_{j l}=g^{i j} \nabla_{i} T_{j l}^{\text {Maxwell }},
$$

since $g^{m l} g^{n k} \nabla_{m} \nabla_{n} B_{j l k}=-(2 / N) \nabla_{j} \nabla_{k}\left(f^{k} / N\right)=0$ in view of Eq. (43).

In other words, the conservation law (50) is given by the usual Maxwell energy-momentum tensor $T_{j l}^{\text {Maxwell }}$ and $N^{2}$ plays the role of the Newton constant $G_{N}$ :

$$
\frac{N^{2}}{2}=\frac{8 \pi G_{N}}{c^{4}} .
$$

The generalized Einstein and Maxwell equations (41)-(48) will be modified further upon inclusion of $s$-derivative terms. Equation (51), however, holds regardless of whether the $s$-derivative terms are included or not. One has to point out here that in the setup of Thiry [5], and in [14], $G_{N} \simeq \phi^{2}$, where $\phi$ satisfies Eq. (49). This implies that a constant solution for $\phi$ and, consequently, $G_{N}$ is possible only when the unphysical constraint $F^{i k} F_{i k}=0$ is satisfied.

In contrast, in the dual setup, a constant solution is possible. However, $N$ (together with $A_{i}$ and $g_{i j}$ ) is a solution to the system of equations (41), (43), and (44) and, in general, does not need to be a constant. Then it plays the role of a dilaton field.

To illustrate this, consider the standard cosmological metric [11] with $E_{\mu}^{\nu}=\delta_{\mu}^{\nu}$ :

$$
\begin{aligned}
d s_{(5)}^{2}= & -s^{2} d t^{2}+t^{2 / \alpha} s^{2 /(1-\alpha)}\left(d r^{2}+r^{2} d \Omega^{2}\right) \\
& +\alpha^{2}(1-\alpha)^{-2} t^{2} d s^{2} .
\end{aligned}
$$

Changing variables by $r \rightarrow s^{\gamma} e^{\beta r}$ with $\gamma=-(1 / 2)(1$ $+\alpha) /(1-\alpha)$ and $t^{1 / \alpha} \rightarrow a(t)$, we get

$$
\begin{aligned}
d s_{(5)}^{2}= & -s^{2} \alpha^{2}[a(t)]^{2 \alpha-2} \dot{a}^{2}(t) d t^{2}+2 A_{r} d r d s \\
& +s a^{2}(t) e^{2 \beta r}\left(\beta^{2} d r^{2}+d \Omega^{2}\right)+\phi d s^{2},
\end{aligned}
$$

where $A_{r}=\gamma \beta a^{2}(t) e^{2 \beta r}, \quad A_{t}=A_{\varphi}=A_{\theta}=0, \quad$ and $\phi$ $=\left(\gamma^{2} / s\right) a^{2}(t) e^{2 \beta r}+\alpha^{2}(1-\alpha)^{-2} a^{2 \alpha}(t)$.

We take $\beta$ to be a negative constant, so that the field $A_{r}$ will fall off toward infinity. Since $a(t)$ describes the inflation, we note that the field $A_{r}$ expands as $a^{2}(t)$. The dilaton (which models Newton's constant) varies as

$$
N^{2}=(1-\alpha)^{2} \alpha^{-2}[a(t)]^{-2 \alpha}
$$

Thus,

$$
\frac{\dot{G}}{G}=-2 \alpha \frac{\dot{a}}{a}=-2 \alpha H,
$$

where $H$ is Hubble's constant. Observational limits [7] put $\alpha<10^{-3}$.

One should note that the four-dimensional metric is now $s$ dependent, but this does not pose a problem in the slicing formulation. Only the term $\partial_{s} g_{j l}$ from the extrinsic curvature (33) should be recovered.

Finally, we note that the general solution for the dilaton field can be written as

$$
N^{2}=\frac{\left(\operatorname{det} g_{i k}\right)\left(\operatorname{det} E_{\nu}^{\mu}\right)^{2}}{\operatorname{det} G_{\mu \nu}},
$$

for a solution $G_{\mu \nu}$ of Eq. (36) and embedding specified with $E_{\nu}^{\mu}$.

To recapitulate, we have found plausible generalizations of the Einstein-Maxwell equations and explained the origin of the constant solution for the dilaton (representing Newton's constant $G_{N}$ ) as well as the possibilities for modeling nonconstant solutions for different cosmologies (representing time-varying $G_{N}$ ) in relation to the gauge freedom of our model.

We thank Vesselin Gueorguiev, Brian Dolan, Brien Nolan, and Siddhartha Sen for useful discussions and comments. 
[1] Th. Kaluza, Sitzungsber. K. Preuss. Akad. Wiss. 69, 966 (1921).

[2] O. Klein, Z. Phys. 37, 895 (1926).

[3] Modern Kaluza-Klein Theories, edited by T. Appelquist, A. Chodos, and P.G.O. Freund (Addison-Wesley, Reading, MA, 1987).

[4] P. Jordan and C. Müller, Z. Naturforsch. A 2, 1 (1947).

[5] Y. Thiry, C. R. Acad. Sci. Hebd Seances Acad. Sci. D 226, 216 (1948).

[6] P.A.M. Dirac, Proc. R. Soc. London A165, 199 (1938).

[7] Th. Damour, G.W. Gibbons, and J.H. Taylor, Phys. Rev. Lett. 61, 1151 (1988); Th. Damour, G.W. Gibbons, and C. Gundlach, ibid. 64, 123 (1990).

[8] J. Ponce de Leon, Class. Quantum Grav. 20, 5321 (2003).

[9] E. Garcia-Berro, Yu.A. Kubyshin, and P. Loren-Aguilar, gr-qc/0302006.

[10] J.-P. Uzan, Rev. Mod. Phys. 75, 403 (2003).

[11] J. Ponce de Leon, Gen. Relativ. Gravit. 20, 539 (1988).

[12] S.S. Seahra and Paul S. Wesson, Class. Quantum Grav. 20, 1321 (2003).
[13] F. Dahia and C. Romero, J. Math. Phys. 43, 3097 (2002).

[14] X. Yang, Y. Ma, J. Shao, and W. Zhou, Phys. Rev. D 68, 024006 (2003).

[15] S. Boersma and T. Dray, Gen. Relativ. Gravit. 27, 319 (1995).

[16] A.L. Zelmanov, Dokl. Akad. Nauk SSSR 107, 815 (1956); 227, 78 (1976).

[17] A. Einstein and P. Bergmann, Ann. Math. 39, 683 (1938); also in [3].

[18] Z. Perjes, Nucl. Phys. B403, 809 (1993).

[19] G. Fodor and Z. Perjes, Gen. Relativ. Gravit. 26, 759 (1994).

[20] S. Boersma and T. Dray, J. Math. Phys. 36, 1378 (1995).

[21] S. Boersma and T. Dray, J. Math. Phys. 36, 1394 (1995).

[22] R. Arnowitt, S. Deser, and C.W. Misner, in Gravitation: An Introduction to Current Research, edited by L. Witten (Wiley, New York, 1962).

[23] T. Shiromizu, K. Maeda, and M. Sasaki, Phys. Rev. D 62, 024012 (2000).

[24] R.M. Wald, General Relativity (University of Chicago Press, Chicago, 1984). 